\title{
Scaling Uncertainty in Visual Perception and Estimation Tasks
}

\author{
Eleanor B. Schille-Hudson' ${ }^{1}$ (erbrower@iu.edu), David Landy ${ }^{1,2}$ (dhlandy@gmail.com) \\ ${ }^{1}$ Department of Psychological and Brain Sciences, Indiana University; ${ }^{2}$ Netflix
}

\begin{abstract}
Demographic perception - the perception of social quantities of geopolitical scale and social significance - has been extensively studied in cognitive and political science (Citrin \& Sides, 2008; Gilens, 2001; Herda, 2013). Regular patterns of over- and under-estimation emerge. Americans greatly overestimate, for instance, the proportion of citizens that identify as gay or Muslim, while underestimating those that are Christian. While these errors have been attributed to social factors such as fear of specific minorities (Gallagher, 2003; Wong, 2007), other work has suggested that these patterns result from the psychophysics of the perception of proportions (Landy, Guay \& Marghetis 2018). A Bayesian formulation suggests that biases in the estimation of both social proportions and simple visual properties result from a common source: 'hedging' uncertain information toward a prior. Here we present a novel lab paradigm and two experiments that manipulate uncertainty in a simple (dot estimation) task, verifying the core assumptions of the Bayesian approach.
\end{abstract}

Keywords: visual perception, uncertainty, scaling, estimation, psychophysics, jsPsych

\section{Introduction}

It is a well documented phenomenon in political science and social psychology that when asked to give an estimate about a demographic group, people are often vastly wrong (Citrin \& Sides, 2008; Gilens, 2001; Herda, 2013). For example, a recent Gallup poll found that U.S. residents estimated that more than $20 \%$ of the population identifies as LGBT (Newport, 2015), while the true proportion is closer to $3 \%$ (Gates \& Newport, 2012). In the past few years, the finding that people are so inaccurate in their estimates has been picked up by popular and news media and panned with some urgency. For example The Guardian ran a headline, "Today's Key Fact: You Are probably Wrong About Almost Everything" (October 29, 2014) and Slate featured "Americans Drastically Overestimate How Many Unauthorized Immigrants Are in the Country, and They Don't Want to Know the Truth" (January 9, 2012). Often, the concern and the urgency behind these findings comes from the worry that people are wrong in their estimates of demographic perceptions because of motivated reasoning or underlying bias (Wong, 2007).

This concern is understandable, as the estimation of demographic proportions matters in part because the surveyor can use it as a window into the estimator's underlying motivations and beliefs. However, to characterize people's mis-estimations of demographic proportions purely as evidence of bias is misleading. As
Landy, Guay \& Marghetis (2018) and Brower \& Landy (2018) observe, claims that mis-estimates are the result of bias alone neglect years of psychophysical research which find nearly identical patterns of mis-estimation in contexts where underlying bias is impossible. The pattern of mis-estimation, specifically overestimation of small proportions and underestimation of large proportions shows up in experiments in which people estimated numerical magnitudes (Barth \& Paladino, 2011), dots on a screen (Varey, Mellers, \& Birnbaum, 1990), and the proportion of letters in a string that are "a" (Erlick, 1964). Landy et al. (2018) and Brower \& Landy (2018) attribute this pattern of errors to uncertainty. Landy et al. (2018) propose a model which accounts for the over-under pattern of mis-estimation using uncertainty. Brower \& Landy (2018) propose a model which differentiates uncertainty from bias.

Using international data from the research survey group Ipsos MORI, Brower \& Landy (2018) created a model that derived previous models of proportion estimation in psychophysics, by assuming a Bayesian notion of hedging in towards a prior. This model differentiated bias and uncertainty by pooling across countries within different survey questions. Landy et al. (2018) and Brower \& Landy (2018) posit that when people's estimates are affected by uncertainty rather than bias, their estimates hedge in towards a prior, often $50 \%$ (the center of the range). Rationally, the less information one has, the more one should hedge in toward that prior. Brower \& Landy (2018) claim that bias distorts the responses of people in specific ways, namely by disproportionately pulling the curve of responses up or down depending on whether the bias in question tends toward over- or under-representation.

Despite accounting for both bias and uncertainty in their model, Landy et al. (2018) as well as Brower \& Landy (2018) base their modeling of uncertainty on reasonable assumptions rather than on direct experimental evidence.

Previous work has investigated estimations in bounded spaces, including proportions and percentages. For example Gonzalez \& Wu (1999), Hollands \& Dyre (2000), Varey et al. (1990), Erlick (1964), Zax et al. (2019) have all conducted psychophysical experiments in which participants' responses fall along a similar inverse s-shaped curve (see Figure 1). In traditional psychophysical cases, these analyses were sometimes derived from Steven's law (Spence, 1990; Hollands \& Dyre, 2000; Barth \& Paladino, 2011). However, none of this work has experimentally manipulated uncertainty in the true proportion, nor has it attributed central hedging to uncertainty. 
Another line of work has emphasized uncertainty. (Huttenlocher, Hedges \& Duncan, 1991). This work, however, has centrally examined uncertainty in memory rather than in perception, and has typically explored absolute values rather than proportions. This line of research has found roughly linear hedging toward a central position, in line with a rational Bayesian model. In this case, uncertainty in memory has largely been manipulated through varying memory load and time delays (Huttenlocher, et al, 1991), or intrinsic memory capacity (Crawford, Landy, and Salthouse, 2017).

The Bayesian application of uncertainty to demographic perception posits that uncertainty leads to the misestimation of minorities and majorities outlined above. However, this kind of uncertainty is principally a result of sampling limitations: the average person simply cannot accurately assess the population of, for instance, the United States.

In this paper, we attempt to experimentally manipulate uncertainty in a proportion estimation context using differential visibility. We ask people to estimate the proportion of circles of a given color, when they know that many circles were hidden behind an occluder. This replicates the central mechanism we and others posit to cause massive error in demographic estimation (limited sampling of a large hidden population) in a laboratory context.
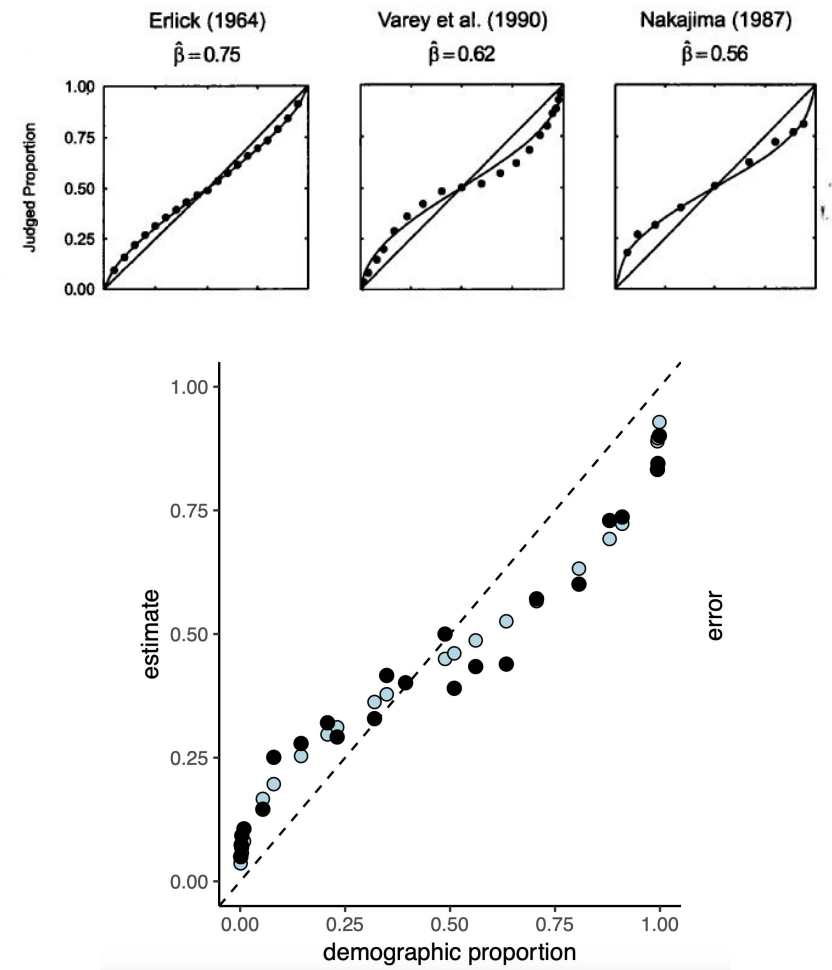

Figure 1: Top (reprinted from Hollands \& Dyre, 2000):

Classic examples of the 'over-under' pattern from

laboratory experiments in psychophysics. Bottom (reprinted from Landy et al, 2018)

\section{Experiment 1}

\section{Methods}

We recruited adults $(N=89)$ from the United States through Amazon Mechanical Turk, an online labor market that has been used previously for online studies. Each subject participated in return for $\$ 4$.

Participants were presented with an online percentage estimation task created using jsPsych. They were presented with a screen full of 100 circles (Fig. 2) However, some of the circles were occluded behind a black rectangle (Fig. 3a). As Figs. 2 and 3 show, there were two kinds of circles: blue and orange ones. The blue and orange circles were of the same saturation/brightness, but these colors were chosen to be relatively color-blind friendly. Every trial displayed a new distribution of dots and trials were independent. After each trial, participants were asked to estimate what percentage of the circles on the whole screen, even the hidden parts were blue. In Experiment 1, in every trial, the circles were in motion. They "bounced" around the screen, off of one another and off of the window borders, moving both out from and behind the occluders as they bounced.

Experiment 1 had a $2 \times 2$ design, with two trial lengths and two sizes of occluding borders (Figs. 3a, 3b). Trials were either one second or ten seconds in duration and had either a small occluding border (Fig. 3a) or a large one (Fig. 3b).

At the end of each trial, participants were instructed to enter a number between 0 and 100 as their estimate of the total percentage of blue circles on the screen. There were a total of 100 circles on the screen and 20 unique distributions of blue circles shown, ranging from 0.01 to 0.99 , with heavier representation of the bottom and top deciles. Each distribution was shown twice for a total of 160 trials.

In the first round of data collection $(N=69)$, the true proportion of blue circles on the screen, as well as the participants' proportion estimates were recorded. In the second round of data collection $(N=20)$, an additional frame-by-frame $\log$ of which circles were visible was also recorded. Circles were also tagged as blue or orange, so that the observed proportion of blue visible circles could be recorded. This was to determine whether the different samples of circles participants saw when parts of the screen were occluded created enough noise to affect their estimates. They did not, and so all participant trials were analyzed together.

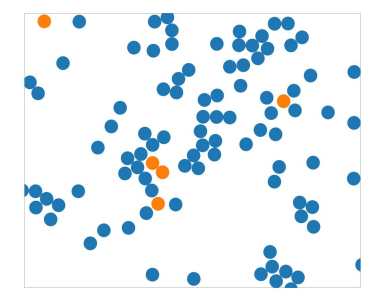

Figure 2: Example screen of blue and orange circles 

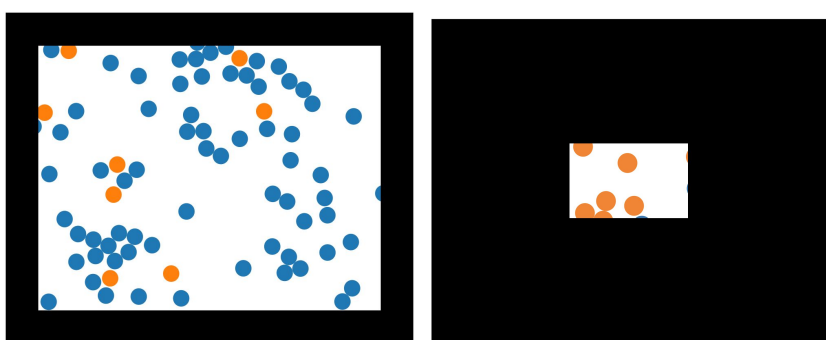

Figure 3a \& 3b: 3a shows a screen of circles with a small occluder, 3 b shows a screen with a large occluder

\section{Analysis}

Previous analyses of demographic response times suggests that a log-odds model would fit proportion judgments well (Gonzalez \& Wu, 1994; Landy et al. 2018; Brower \& Landy, 2018). We fit each subject in each condition (occluder size x exposure time) to a log-linear model:

$$
\psi(p)=\frac{\delta^{1-\gamma} p^{\gamma}}{\delta^{1-\gamma} p^{\gamma}+(1-p)^{\gamma}}
$$

This model has two relevant parameters (plus an error term): $\gamma$ and $\delta . \quad \gamma$ (hedging) indicates the degree of overestimation; while $\delta$ (offset) measures the overall error level. Roughly, the lower the value of delta, the more underestimation overall; higher values of delta correspond to overestimation across the range (delta is usually reported as a multiplicative value: here the value we analyse corresponds to $\log$ (delta) in other approaches). The version of the log odds model we employ here is based on a Bayesian derivation $\gamma$ reflects the relative use of prior and posterior information, and is theoretically constrained to be between 0 (full use of prior) and 1 (relying just on the current stimulus). In this same model, the offset results from a combination of the two parameters: in the probability (rather than $\log$ odds) space, $\delta$ is raised to the $(1-\gamma)$ (see Landy et al., 2018 for a derivation). We follow this published pattern, but checked that none of our results change in tone if rendered in the more classical psychophysical formulation.

Evaluating the log-odds mode properly requires extreme values, less than 1\% and more than 99\% (see Figure 1). Without these extremes, the model is very close to linear. Our intention was not to test the log-odds model against linear regression per se, but rather to use it as an analytical framework for understanding how occluders affected a general inward hedging in proportion estimation.

Our primary hypotheses were that (1) people would hedge in toward the mean in-line with the classic 'over-under' inverse-S curve, and that (2) $\gamma$ would be lower (e.g., hedging would be larger) when the occluder was large and time was short.

\section{Results}

Overall, the log-odds model was successful in capturing the pattern of most participants' responses: the median correlation between the model and individual participant responses was $r=0.86$ within each participant and test cell. We did not expect to be able to distinguish the log-odds model from a linear model, and we could not: The log likelihood for this model was better than a linear model for $50 \%$ of the participant $\mathrm{x}$ condition data sets. Furthermore, in no case was it substantially better or substantially worse. Since the model fit overall quite well, and prior work supports a log-odds model we used the log-odds model parameters as the unit of subsequent analysis.

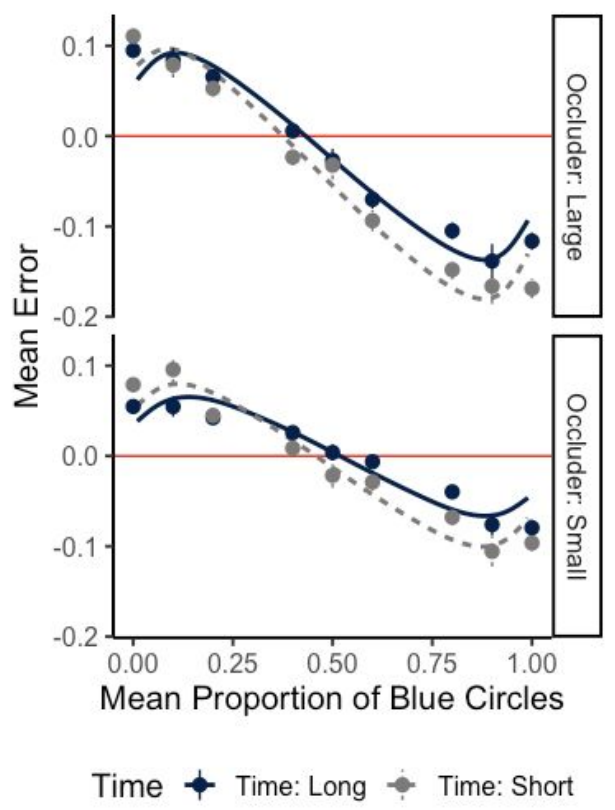

Figure 4: Averaged data across participants illustrating increased hedging (steeper curves) for both shorter time intervals and larger occluders. The dots show mean data, while the curves are averaged model fits.

Table 1: Distribution of parameters for Experiment 1
Condition

Occluder: Large

1s presentation

$0.59 \pm 0.02$

$0.85 \pm 0.03$

10s presentation

$0.64 \pm 0.03$

$0.95 \pm 0.04$

Occluder: Small

1s presentation

$0.71 \pm 0.03$

$0.98 \pm 0.02$

10s presentation

$1.04 \pm 0.03$
$0.78 \pm 0.02$

Note: Errors are standard errors around the mean. 


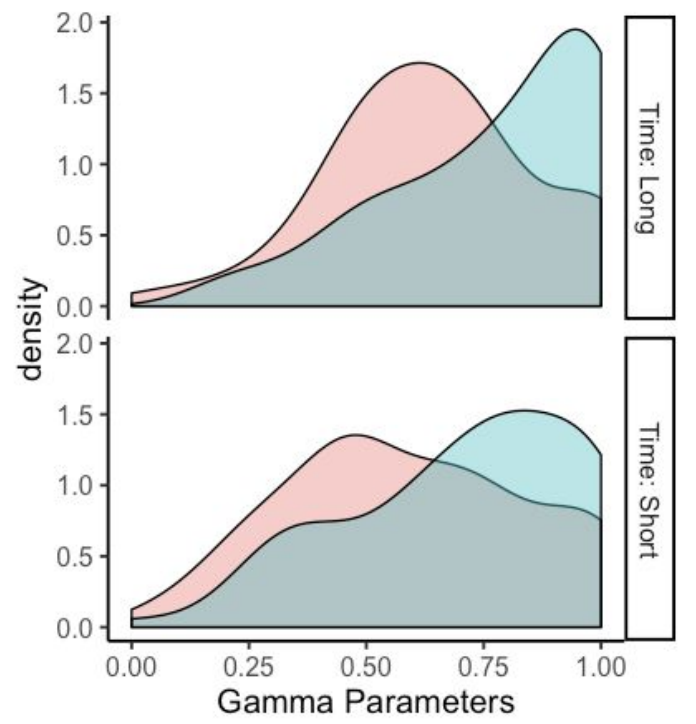

occluder $\square$ Occluder: Large $\square$ Occluder: Small

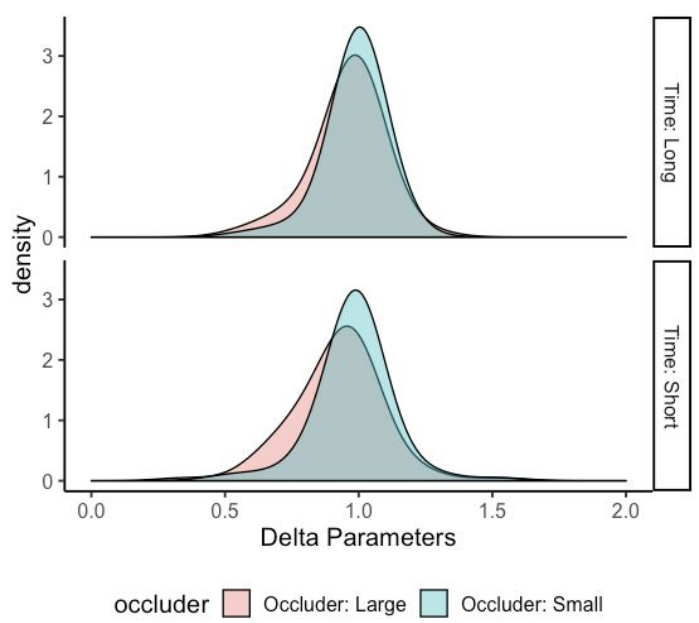

Figure 5: Top: probability density of Gamma parameters Bottom: probability density of Delta parameters. Both are broken down by stimulus duration and occluder size.

To evaluate significance, we fit nested random effects models to the gamma and delta parameters, including main effects for both time and occluder size. The model that included a random effect for both occluder size and time $(\mathrm{BIC}=-59 ; \operatorname{logLike}=56)$ provided a substantially and significantly ( $p<0.001$ by a likelihood ratio test) improved over the base model $(\mathrm{BIC}=-49, \operatorname{logLik}=33)$. The model including just a time parameter $(\mathrm{BIC}=-34 ; \log \mathrm{Lik}=35) \mathrm{did}$ not provide a compelling alternative nor did just the occluder model $(\mathrm{BIC}=-59, \operatorname{logLik}=47)$-though the BIC value for this model was essentially identical to that of the full model.
Overall values of $\delta$ were extremely close to 1 , suggesting that neither overestimation nor underestimation in log odds space dominates. The mean $\delta$ was 0.96 . This was significantly impacted by both occluder size and time (Full model $\mathrm{BIC}=-556 ; \operatorname{logLik}=608$ The next best model had just an effect of occluder $(\mathrm{BIC}=-510$; $\log \mathrm{Lik}=263$ ). Again, taken individually, the impact of occluder significantly improved the model fit compared to baseline by a likelihood ratio test $(\mathrm{BIC}=-536$; $\log \mathrm{Lik}=285)$, but the impact of time alone did not $(\mathrm{BIC}=-495 ; \log \mathrm{Lik}=265)$. In general, both shorter times and larger occluders led to underestimation of the blue dot proportion.

It is worth noting that unlike classic psychophysical models (e.g., Spence 1990), the Bayesian log-odds model used here intimately connects inward hedging (the $\gamma$ parameter) with the offset (see the equation). In particular, steeper slopes lead to offsets further from the middle value $(50 \%)$. The impact of time and occlusion to $\delta$ is in addition to that general pattern.

\section{Discussion}

In the results from Experiment 1, we do indeed see the typical over-under pattern of errors. Participants overestimated small proportions of blue circles and underestimated large proportions of blue circles. As hypothesized, participants hedged in towards a uniform prior more when they responded to a shorter trial. They also hedged more with larger occluders, as hypothesized. These results confirm that uncertainty can be scaled with time of presentation and limited visibility.

Experiment 1 also showed a small but highly significant pattern in which people had lower offsets - they underestimated more-when the occluders were large and time was limited. This result was not expected, and may result from specific strategies employed by some individuals that focus on the "numerator" or focal group (such as attempting to count individual blue circles).

In Experiment 2, we verify two further links to classical psychophysical results. First, we entirely remove the occluders. Under the uncertainty hypothesis, the inward hedging should be reduced but not eliminated compared to Experiment 1 (assuming people are not literally counting the number of blue dots, which would be difficult in short presentations). Second, we evaluate the impact of the most significant design decision of Experiment 1: the moving dots. In Experiment 2, we manipulate the dot motion, comparing moving to static dots. We have no strong reason to believe that moving dots would operate differently from static dots: however, while having dots move does not reduce access to information around them the way that occluders do, it may well interfere with information processing. 


\section{Experiment 2}

\section{Methods}

Experiment 2 was largely a replication of Experiment 1 with a few modifications. The occluders were removed, so that participants had full information and could see all the circles on the screen for the entire duration of the trial. There were still two different trial lengths, one second and ten seconds. However, there were now two different types of trials: static and moving. In half of the trials, participants were presented with the same screen full of moving circles, but in the other half, the circles did not move. We made this change to determine whether the motion of the circles was additionally scaling uncertainty. We again recruited adults $(N=59)$ from the United States through Amazon Mechanical Turk. We used the same 20 distributions of blue dots in each trial, but participants only saw each distribution once, for a total of 80 trials.

\section{Analysis}

The analysis matched that of Experiment 1. Again, maximum likelihood was used to fit each participant $\mathrm{x}$ condition combination, and the resulting parameters were analyzed.

\section{Results}

Again, the log-odds model was adequately related to most responses. The mean correlation was $r=0.89$ between individual responses and the model.

Though results were similar (see Figure 6), participants showed less inward hedging when the circles were static: the best-fitting random-effects model had only an effect of trial type $(\mathrm{BIC}=-129$; $\operatorname{logLik}=81)$; this was better than the baseline model $(\mathrm{BIC}=-137$, logLik $=77)$; the full model including time did not significantly improve it by a likelihood ratio test $(\mathrm{BIC}=-112 ; \operatorname{logLik}=81)$.

Table 2: Distribution of parameters for Experiment 2

\begin{tabular}{lll} 
Condition & $\gamma$ & $\delta$ \\
\hline Circles: Moving & & \\
1s presentation & $0.84 \pm 0.03$ & $1.05 \pm 0.04$ \\
10s presentation & $0.82 \pm 0.03$ & $1.19 \pm 0.01$ \\
Circles: Static & & \\
1s presentation & $0.84 \pm 0.03$ & $0.99 \pm 0.04$ \\
10s presentation & $0.85 \pm 0.03$ & $1.08 \pm 0.04$
\end{tabular}

Note: Errors are standard errors around the mean.

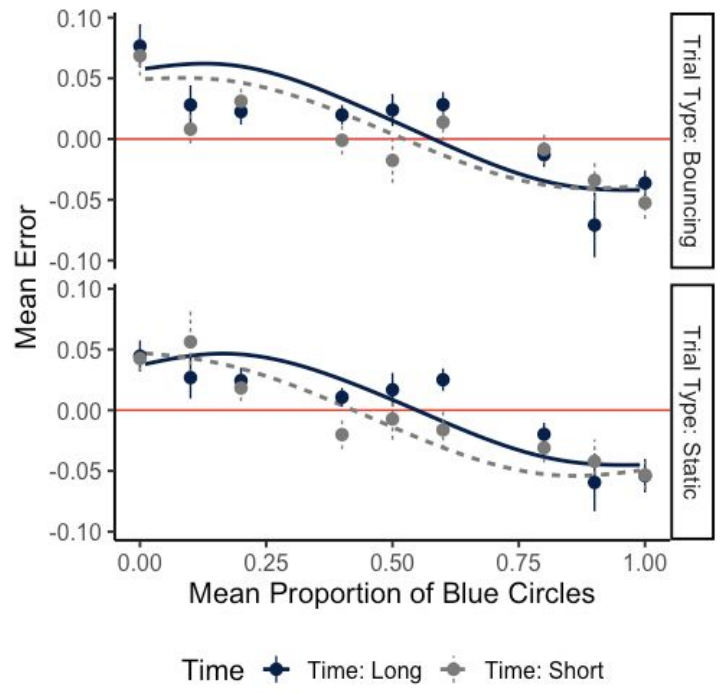

Figure 6: Averaged data across participants in Experiment 2. The dots show mean data, while the curves are averaged model fits. People hedged slightly more when the circles moved, and underestimated (lower offsets) when time was short or the circles were static.

Though present, the effect of motion on hedging was modest, and in both conditions the inward hedging was less pronounced in the full-visibility trials (compare Table 2 to Table 1). Although presentation time did not have a significant impact on inward hedging, it did significantly affect overall over-/under-estimation (note the separation between the blue and gray lines in Figure 6). In fact, the model including effects of both trial type and time of presentation on offset was the best fitting $(\mathrm{BIC}=-427$; logLik = 238), and significantly improved on the baseline $(\mathrm{BIC}=-389 ; \operatorname{logLik}=203)$, time-only $(\mathrm{BIC}=-412 ; \operatorname{logLik}$ $=222)$ and trial type only $(\mathrm{BIC}=-374 ; \operatorname{logLik}=203)$ models.

In pattern, having more time led to more overestimation (in these trials, blue circles were overestimated slightly, not underestimated); blue circles were also overestimated more in moving-circle trials.

\section{General Discussion}

Generally, the results supported our predictions: hedging was more pronounced when uncertainty was limited by a variety of factors: partial occlusion (Experiment 1), time of presentation (Experiments 1 and 2), and movement (Experiment 2). These results strongly confirm uncertainty-based interpretations of the inverse-s pattern in demographic estimation (Landy et al, 2018; Brower \& Landy, 2018; c.f Zaromb et al., 2018), and suggests that publications that look first to social factors without accounting for psychophysical ones risk misinterpreting results (Gallagher, 2003; Wong, 2007). 
Across two experiments, we also found significant shifts to overall baselines: the offset $(\delta)$ parameters were lower under shorter time intervals, with more occlusion, and with static presentations (it's is worth emphasizing that the same effect is found when the model is formulated with a classical $\delta$ (see Gonzalez \& Wu, 1999). There is no clear pattern to these effects: they do not follow the differential information the way the hedging parameters do. Generally speaking, factors that affect offsets are less well-studied and less well-understood than those that affect hedging. Nonetheless, in some contexts these effects can be quite large and of broad social importance (e.g., Brower \& Landy, 2018); understanding them is probably important. In this case, we hypothesize that the differences may have resulted from differential strategies. Static presentation and smaller windows support counting strategies for instance, and these may be associated with more underestimation. Alternatively, there may be baseline differences in visual salience between blue and orange (these colors were not counterbalanced; blue was always the foreground color) that manifest differently in different visual conditions. However, these are post-hoc guesses: this important topic remains unexplored in our work, as well. In future work, we intend to create trials with more circles so that true proportions smaller than .01 and larger than .99 could be tested. We also are interested in the effect of more (and counterbalanced) colors and shapes on participants' hedging behavior.

The occlusion results are of primary practical value: Unlike real-world demographic proportions, information in the lab can be precisely controlled. The main purpose of this work is in some ways methodological: we are establishing that inverse s-shapes can be manipulated by precise control of visual information. In future work, we or other researchers can use this method to test Bayesian and other derivations of the inverse-s pattern (e.g., Zax et al, 2019; Hollands \& Dyre, 2000; Landy et al, 2018; Petzschner, 2012). Applications of psychophysics to issues of broad social importance are rare, but we believe that in this case, hope is justified.

\section{References}

Barth, H. C., \& Paladino, A. M. (2011). The development of numerical estimation: Evidence against a representational shift. Developmental Science, 14, 125-135. doi: 10.1111/j.1467-7687.2010.00962.x

Brower, E., Landy, D., (2018). Bias in the Self-Knowledge of Global Communities. Proceedings of the $40^{\text {th }}$ Cognitive Science Society. Madison, WI

Citrin, J., \& Sides, J. (2008). Immigration and the Imagined Community in Europe and the United States. Political Studies, 56, 33-56.

Crawford, L. E., Landy, D., \& Salthouse, T. A. (2016). Spatial working memory capacity predicts bias in estimates of location. Journal of Experimental Psychology: Learning, Memory, and Cognition, 42(9), 1434.

Gallagher, C. (2003). Miscounting Race: Explaining Whites' Misperceptions of Racial Group Size. Sociological Perspectives, 46(3), 381-396.

Gates, G. J., \& Newport, F. (2012). Special report: 3.4\% of U.S. adults identify as LGBT. Gallup. Retrieved from www.gallup.com/poll/158066/special-report-adults-identi fy-lgbt.aspx

Gilens, M. (2001). Political Ignorance and Collective Policy Preferences. The American Political Science Review, 95(2), 379-396.

Gonzalez, R., \& Wu, G. (1999). On the Shape of the Probability Weighting Function. Cognitive Psychology, 38, 129-166

Herda, D. (2013). Too Many Immigrants?: Examining Alternative Forms of Immigrant Population Innumeracy. Sociological Perspectives, 56(2), 213-240.

Hollands, J. G., \& Dyre, B. P. (2000). Bias in proportion judgments: The cyclical power model. Psychological Review, 107(3), 500-524.

Huttenlocher, J., Hedges, L. V., \& Duncan, S. (1991). Categories and particulars: Prototype effects in estimating spatial location. Psychological review, 98(3), 352.

Huttenlocher, J., Hedges, L. V., \& Duncan, S. (1991). Categories and particulars: Prototype effects in estimating spatial location. Psychological Review, 98(3), 352-376.

Landy, D., Guay, B., \& Marghetis, T. (2018). Bias and ignorance in demographic perception. Psychonomic Bulletin \& Review, 25, 1606-1618.

Newport, F. (2015). Americans greatly overestimate percent gay, lesbian in U.S. Gallup. Retrieved from www.gallup.com/poll/183383/americans-greatly-overesti mate-percent-gay-lesbian.aspx

Petzschner, F. (2012). Magnitude Estimations in Humans. Ludwig-Maximilians-Universität, München.

Spence, I. (1990). Visual psychophysics of simple graphical elements. Journal of Experimental Psychology: Human Perception and Performance, 16(4), 683.

Wong, C. (2007). "Little" and "Big" Pictures in our Heads: Race, Local Context, and Innumeracy about Racial Groups in the United States. Public Opinion Quarterly, 71(3), 392-412.

Zaromb, F. M., Liu, J. H., Páez, D., Hanke, K., Putnam, A. L., \& Roediger III, H. L. (2018). We made history: Citizens of 35 countries overestimate their Nation's role in world history. Journal of Applied Research in Memory and Cognition, 7(4), 521-528.

Zax, A., Williams, K., Patalano, A. L., Slusser, E., Cordes, S., \& Barth, H. (2019). What do biased estimates tell us about cognitive processing? Spatial judgments as proportion estimation. Journal of Cognition and Development, 20(5), 702-728. 